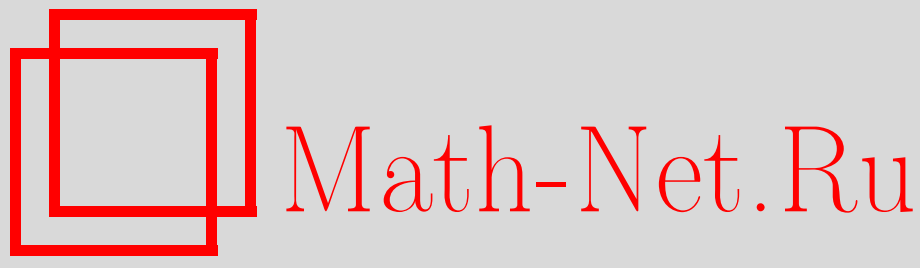

Общероссийский математический портал

В. А. Юрко, О дифференциальных операторах высших порядков с особенностью внутри интервала, Матем. заметки, 2002, том 71, выпуск 1, 152-156

DOI: https://doi.org/10.4213/mzm640

Использование Общероссийского математического портала Math-Net.Ru подразумевает, что вы прочитали и согласны с пользовательским соглашением http://www.mathnet.ru/rus/agreement 
Параметры загрузки:

IP : 54.197 .217 .227

26 апреля 2023 г., 14:47:03

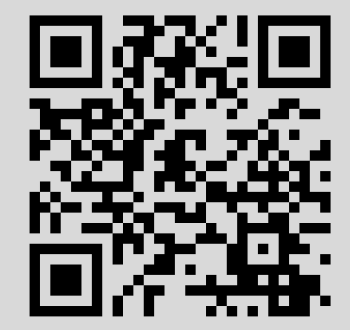




\section{О ДИФФЕРЕНЦИАЛЬНЫХ ОПЕРАТОРАХ ВЫСШИХ ПОРЯДКОВ С ОСОБЕННОСТЬЮ ВНУТРИ ИНТЕРВАЛА}

\section{В. А. Юрко}

1. Рассмотрим дифференциальное уравнение

$$
\ell y(x):=y^{(n)}(x)+\sum_{j=0}^{n-2}\left(\frac{\nu_{j}}{(x-a)^{n-j}}+q_{j}(x)\right) y^{(j)}(x)=\lambda y(x), \quad 0<x<T
$$

с неинтегрируемой регулярной особенностью во внутренней точке $a \in(0, T)$. Здесь $q_{j}(x)-$ комплекснозначные функции, $\nu_{j}$ - комплексные числа. Пусть $\mu_{1}, \ldots, \mu_{n}$ - корни характеристического многочлена

$$
\delta(\mu)=\sum_{j=0}^{n} \nu_{j} \prod_{k=0}^{j-1}(\mu-k), \quad \nu_{n}:=1, \quad \nu_{n-1}:=0
$$

и пусть для определенности $n=2 m, \mu_{k}-\mu_{j} \neq s n(s \in \mathbb{Z}) ; \operatorname{Re} \mu_{1}<\cdots<\operatorname{Re} \mu_{n}, \mu_{k} \neq 0,1,2, \ldots$, $n-3$ (остальные случаи вносят небольшие изменения). Обозначим $\theta=\operatorname{Re}\left(\mu_{n}-\mu_{1}\right)$ и предположим, что функции $q_{j}^{(\nu)}(x), \nu=0, \ldots, j-1$, абсолютно непрерывны на $[0, a-\varepsilon]$ и $[a+\varepsilon, T]$ при всех $\varepsilon \in(0, \min (a, T-a))$ и $q_{j}^{(\nu)}(x)|x-a|^{n-1-\theta-j+\nu} \in L(0, T), \nu=0, \ldots, j$.

В статье исследуется несамосопряженная краевая задача $\mathscr{L}$ для дифференциального уравнения (1) с краевыми условиями $y^{(\nu-1)}(0)=y^{(\nu-1)}(T)=0, \nu=1, \ldots, m$, и дополнительными условиями склейки решений в окрестности особой точки $x=a$, порожденными матрицей перехода

$$
A=\left[a_{k j}\right]_{k, j=1, \ldots, n}
$$

которая связывает решения уравнения (1) слева и справа от особой точки (см. ниже).

Работа выполнена при поддержке Российского фонда фундаментальных исследований, грант № 00-01-00741.

(C) В. А. Юрко 
Дифференциальные уравнения с особенностями внутри интервала возникают в различных разделах математики и приложениях. К уравнению (1) также сводится широкий класс дифференциальных уравнений с точками поворота. При этом различные задачи приводят к различным условиям склейки, что делает актуальным изучение краевых задач с достаточно общими условиями склейки, порожденными различными матрицами перехода $A$. Отметим, что прямые и обратные задачи спектрального анализа для дифференциальных уравнений высших порядков с интегрируемыми коэффициентами и более общие классы операторов достаточно полно изучены (см. [1]-[8] и литературу в них). Случай, когда особая точка лежит на конце интервала, рассматривался в [9], [10]. Наличие особой точки внутри интервала вносит существенные качественные изменения в исследование краевой задачи. В данной статье изучаются свойства спектра краевой задачи $\mathscr{L}$ и приводятся теоремы о полноте и о разложении по собственным и присоединенным функциям. Отметим, что некоторые аспекты спектральной теории для уравнения (1) при $n=2$ исследовались в [11], [12].

2. Пусть $\lambda=\rho^{n}$. Рассмотрим функции

$$
C_{j}(x, \lambda)=(x-a)^{\mu_{j}} \sum_{k=0}^{\infty} c_{j k}(\rho(x-a))^{n k}, \quad j=1, \ldots, n
$$

где

$$
c_{j k}=c_{j 0}\left(\prod_{s=1}^{k} \delta\left(\mu_{j}+s n\right)\right)^{-1}, \quad \prod_{j=1}^{n} c_{j 0}=\left(\operatorname{det}\left[\mu_{j}^{\nu-1}\right]_{j, \nu=1, \ldots, n}\right)^{-1} .
$$

Здесь и в дальнейшем $z^{\mu}=\exp (\mu(\ln |z|+i \arg z)), \arg z \in(-\pi, \pi]$. При $x>a$ и $x<a$ функции $C_{j}(x, \lambda)$ являются решениями "простейшего" уравнения вида $(1)$ с $q_{j}(x) \equiv 0$.

Пусть функции $s_{j}(x, \lambda), j=1, \ldots, n$, являются решениями следующей системы интегральньх уравнений при $x>a$ и $x<a$ :

$$
s_{j}^{(\nu)}(x, \lambda)=C_{j}^{(\nu)}(x, \lambda)-\int_{a}^{x} \frac{\partial^{\nu}}{\partial x^{\nu}} g_{0}(x, t, \lambda)\left(\sum_{p=0}^{n-2} q_{p}(t) s_{j}^{(p)}(t, \lambda)\right) d t, \quad \nu=0, \ldots, n-1,
$$

где

$$
\begin{gathered}
g_{0}(x, t, \lambda)=\sum_{j=1}^{n}(-1)^{n-j} C_{j}(x, \lambda) C_{n-j+1}^{*}(t, \lambda), \\
C_{j}^{*}(x, \lambda)=\operatorname{det}\left[C_{k}^{(\nu)}(x, \lambda)\right]_{\nu=0, \ldots, n-2, k=1, \ldots, n \backslash n-j+1} .
\end{gathered}
$$

При каждом $x \neq a$ функции $s_{j}^{(\nu)}(x, \lambda)$ являются целыми по $\lambda$ порядка $1 / n$. При $x>a$ и $x<a$ функции $s_{j}(x, \lambda)$ образуют фундаментальную систему решений уравнения $(1)$, причем

$$
\operatorname{det}\left[s_{j}^{(\nu-1)}(x, \lambda)\right]_{j, \nu=1, \ldots, n} \equiv 1 .
$$

Пусть задана матрица $A=\left[a_{k j}\right]_{k, j=1, \ldots, n}, \operatorname{det} A \neq 0$, где $a_{k j}$ - комплексные числа. Введем функции $\left\{\sigma_{j}(x, \lambda)\right\}_{j=1, \ldots, n}, x \in J_{ \pm}$, где $J_{ \pm}:=\{x: \pm(x-a)>0\}$, по формуле

$$
\sigma_{j}(x, \lambda)= \begin{cases}s_{j}(x, \lambda), & x \in J_{-}, \\ \sum_{k=1}^{n} a_{k j} s_{k}(x, \lambda), & x \in J_{+} .\end{cases}
$$

Фундаментальная система решений $\left\{\sigma_{j}(x, \lambda)\right\}$ использется для склейки решений уравнения $(1)$ в окрестности особой точки $x=a$. Точнее, будем говорить, что решение $y(x, \lambda)$ уравнения $(1)$ удовлетворяет условиям склейки, порожденными матрицей перехода $A$, если $y(x, \lambda)$ представима в виде

$$
y(x, \lambda)=\sum_{j=1}^{n} \chi_{j}(\lambda) \sigma_{j}(x, \lambda) \quad \text { при всех } x \in J_{-} \cup J_{+},
$$

где коэффициенты $\chi_{j}(\lambda)$ не зависят от $x$. Для определенности в дальнейшем ограничимся наиболее важньм частным случаем, когда $a_{k j}=0$ при $k<j$. 
3. Пусть функции $\varphi_{j}(x, \lambda), j=1, \ldots, n$, являются решениями уравнения (1) при начальных условиях $\varphi_{j}^{(\nu-1)}(0, \lambda)=\delta_{j \nu}, j, \nu=1, \ldots, n\left(\delta_{j \nu}\right.$ - символ Кронекера), и при дополнительных условиях склейки, порожденных матрицей перехода $A$. При каждом $x \neq a$ функции $\varphi_{j}^{(\nu)}(x, \lambda)$, $\nu=0, \ldots, n-1$, являются целыми по $\lambda$ порядка $1 / n$, причем

$$
\operatorname{det}\left[\varphi_{j}^{(\nu-1)}(x, \lambda)\right]_{j, \nu=1, \ldots, n} \equiv \begin{cases}1, & x \in J_{-} \\ \operatorname{det} A, & x \in J_{+}\end{cases}
$$

Обозначим $S_{k_{0}}=\left\{\rho: \arg \rho \in\left(k_{0} \pi / n,\left(k_{0}+1\right) \pi / n\right)\right\}$. В каждом секторе $S_{k_{0}}$ корни $R_{k}, k=1, \ldots, n$, уравнения $R^{n}-1=0$ можно занумеровать так, что

$$
\operatorname{Re}\left(\rho R_{1}\right)<\operatorname{Re}\left(\rho R_{2}\right)<\cdots<\operatorname{Re}\left(\rho R_{n}\right), \quad \rho \in S_{k_{0}} .
$$

Ясно, что $R_{k}=\exp \left(i \pi \omega_{k} / m\right)$, где $\omega_{k}$-некоторая перестановка чисел $0,1, \ldots, n-1$. В дальнейшем $R_{k}^{\mu}:=\exp \left(i \pi \mu \omega_{k} / m\right)$. При $|\rho(x-a)| \geqslant 1, \rho \in \overline{S_{k_{0}}}, \nu=0, \ldots, n-1, j=1, \ldots, n,|\rho| \rightarrow \infty$, имеют место асимптотические формулы

$$
\begin{aligned}
\varphi_{j}^{(\nu)}(x, \lambda)= & \frac{1}{n} \sum_{k=1}^{n}\left(-\rho R_{k}\right)^{\nu+1-j} \exp \left(-\rho R_{k} x\right)[1] a, \quad x \in J_{-}, \\
\varphi_{j}^{(\nu)}(x, \lambda)= & \frac{1}{n} \sum_{l, k=1}^{n}\left(-\rho R_{k}\right)^{1-j}\left(\rho R_{l}\right)^{\nu}\left(\xi_{k l}^{0}+O\left(\rho^{-\delta_{1}}\right)\right) \\
& \times \exp \left(-\rho R_{k} a\right) \exp \left(\rho R_{l}(x-a)\right)[1]_{a}, \quad x \in J_{+},
\end{aligned}
$$

где $\delta_{1}:=\min \left(1, \min _{l} \operatorname{Re}\left(\mu_{l+1}-\mu_{l}\right)\right),[1]_{a}=1+O\left(|\rho(x-a)|^{-1}+|\rho|^{-\delta_{0}}\right), \delta_{0}=\min (1, \theta)$,

$$
\xi_{k j}^{0}=\sum_{s=1}^{n} a_{s s} R_{k}^{\mu_{s}} d_{s j} \exp \left(-i \pi \mu_{s}\right), \quad\left[d_{s j}\right]_{s, j=1, \ldots, n}=\left(\left[R_{k}^{\mu_{j}}\right]_{k, j=1, \ldots, n}\right)^{-1} \text {. }
$$

Обозначим

$$
\xi_{s}^{0}=\operatorname{det}\left[\xi_{k j}^{0}\right]_{k=1, \ldots, s, j=n-s+1, \ldots, n}, \quad s=1, \ldots, n .
$$

В частности, $\xi_{n}^{0}=(-1)^{m} \operatorname{det} A \neq 0$. Предположим, что

$$
\xi_{s}^{0} \neq 0 \quad \text { при } s=1, \ldots, n-1 .
$$

Условие (2) называется условием регулярности склейки. Контрпример в п. 4 показьвает важность условия регулярности склейки для спектрального анализа дифференциальных операторов с особенностью внутри интервала. Отметим, что если $A=E\left(E-\right.$ единичная матрица) и $\nu_{j}=0$, то $\xi_{k j}^{0}=\delta_{k, n-j+1}$, т.е. $\xi_{2 s}^{0}=\xi_{2 s+1}^{0}=(-1)^{s} \neq 0$, и условие регулярности склейки выполняется.

Обозначим

$$
\Delta(\lambda):=\operatorname{det}\left[\varphi_{j}^{(\nu-1)}(T, \lambda)\right]_{j=m+1, \ldots, n, \nu=1, \ldots, m} .
$$

Функция $\Delta(\lambda)$ является целой по $\lambda$ порядка $1 / n$, и ее нули совпадают с собственными значениями $\left\{\lambda_{l}\right\}$ краевой задачи $\mathscr{L}$. Функция $\Delta(\lambda)$ называется характеристической функцией. Положим

$$
\begin{gathered}
I_{k}=\left\{\rho: \arg \rho=\frac{\pi k}{n}\right\}, \quad k=-n, \ldots, n-1, \\
I_{k, h}=\left\{\rho: \operatorname{dist}\left(\rho, I_{k}\right) \leqslant h\right\}, \quad I_{+1}^{h}=\bigcup_{k} I_{2 k, h}, \quad I_{-1}^{h}=\bigcup_{k} I_{2 k-1, h} .
\end{gathered}
$$

Пусть $\hat{I}_{ \pm 1}^{h}-$ образ $I_{ \pm 1}^{h}$ при отображении $\rho \rightarrow \lambda=\rho^{n}$. 
TeOPEMa 1. $\left(i_{1}\right) \Pi p u|\rho| \rightarrow \infty, \rho \in \overline{S_{k_{0}}}$,

$$
\Delta(\lambda)=O\left(\rho^{-m^{2}} \exp \left(\rho\left(R_{m+1}+\cdots+R_{n}\right) T\right)\right) .
$$

(i, ) Существуют $h>0 u C_{h}^{0}>0$ такие, что

$$
|\Delta(\lambda)| \geqslant C_{h}^{0}\left|\rho^{-m^{2}} \exp \left(\rho\left(R_{m+1}+\cdots+R_{n}\right) T\right)\right| \quad n p u \rho \in \overline{S_{k_{0}}} \backslash I_{(-1)^{m}}^{h} .
$$

Собственные значения $\lambda_{l}=\rho_{l}^{n}$ краевой задачи $\mathscr{L}$ лежсат в области $\hat{I}_{(-1)^{m}}^{h}$.

$\left(i_{3}\right)$ Число нулей $N_{\xi}$ функиии $\Delta(\lambda)$ в области $\Pi_{\xi}^{h}:=\left\{\rho \in I_{(-1)^{m}}^{h}:|\rho| \in[\xi, \xi+1]\right\}$ ограничено по $\xi$.

$\left(i_{4}\right)$ Обозначим $G_{\delta}:=\left\{\rho:\left|\rho-\rho_{l}\right| \geqslant \delta\right\}$. Тогда

$$
|\Delta(\lambda)| \geqslant C_{\delta}\left|\rho^{-m^{2}} \exp \left(\rho\left(R_{m+1}+\cdots+R_{n}\right) T\right)\right|, \quad \rho \in \overline{S_{k_{0}}} \cap G_{\delta} .
$$

$\left(i_{5}\right)$ Существуют положительные числа $r_{N} \rightarrow \infty$ такие, что при достаточно малом $\delta>0$ окружности $|\rho|=r_{N}$ лежат в $G_{\delta}$ для всех $N$.

4. Пусть $B_{\alpha, p}=\left\{f(x): f(x)(x-a)^{-\alpha} \in L_{p}(0, T)\right\}$ - банахово пространство с нормой $\|f\|_{\alpha, p}=\left\|f(x)(x-a)^{-\alpha}\right\|_{L_{p}(0, T)}$. Положим $\omega=\operatorname{Re} \mu_{1}$.

Tеорема 2. Система собственных и присоединенных функций краевой задачи $\mathscr{L}$ полна в $B_{\alpha, p}$ npu $1 \leqslant p<\infty, \alpha<\omega+1 / p$.

Приведем контрпример, показывающий существенность условия регулярности склейки (2). Рассмотрим краевую задачу

$$
\begin{gathered}
-y^{\prime \prime}=\lambda y, \quad 0<x<\pi, \quad \lambda=\rho^{2}, \\
y(0)=y(\pi)=0, \quad y^{(k)}(a+0)=(-1)^{k} y^{(k)}(a-0), \quad k=0,1, \quad a=\frac{3 \pi}{4} .
\end{gathered}
$$

Для этой задачи условие (2) не выполняется и

$$
\Delta(\lambda)=\rho^{-1} \sin \rho(2 a-\pi) .
$$

Собственные значения $\lambda_{l}=\rho_{l}^{2}$ задачи (3) суть $\rho_{l}=2 l, l \geqslant 1$, а собственные функции имеют вид

$$
y_{l}(x)= \begin{cases}\sin 2 l x, & x \leqslant \frac{3 \pi}{4} \\ (-1)^{l-1} \sin 2 l x, & x>\frac{3 \pi}{4} .\end{cases}
$$

Система функций $\left\{y_{l}(x)\right\}_{l \geqslant 1}$ неполна в $B_{\alpha, p}$ при $1 \leqslant p<\infty, \alpha<1+1 / p$.

Обозначим $\eta=\max (0,-\omega), \mu_{k}^{*}=n-1-\mu_{n-k+1}, \psi=\mu_{n-1}+\mu_{1}^{*}$. Пусть для определенности $\operatorname{Re} \psi<0$. Построим функцию $G(x, t, \lambda)$ по формуле

$$
G(x, t, \lambda)=\frac{(-1)^{m}}{\Delta(\lambda)} \mid \begin{array}{cccc}
\varphi_{m+1}(x, \lambda) & \ldots & \varphi_{n}(x, \lambda) & g(x, t, \lambda) \\
\varphi_{m+1}(T, \lambda) & \ldots & \varphi_{n}(T, \lambda) & g(T, t, \lambda) \\
\ldots \ldots \ldots \ldots \ldots \ldots \ldots \ldots \ldots \ldots \ldots \ldots \ldots \ldots \ldots \ldots \ldots \ldots \ldots \ldots & \ldots \ldots \ldots
\end{array} \ldots
$$

где $g(x, t, \lambda)=0$ при $x<t$ и

$$
\begin{gathered}
g(x, t, \lambda)=\sum_{k=1}^{n}(-1)^{k-1} \varphi_{n-k+1}(x, \lambda) \varphi_{k}^{*}(t, \lambda), \quad x \geqslant t, \\
\varphi_{k}^{*}(t, \lambda)=\frac{1}{A(t)} \operatorname{det}\left[\varphi_{j}^{(\nu)}(t, \lambda)\right]_{\nu=0, \ldots, n-2, j=1, \ldots, n \backslash n-k+1}, \quad A(t)= \begin{cases}1, & t \in J_{-}, \\
\operatorname{det} A, & t \in J_{+} .\end{cases}
\end{gathered}
$$


ТЕОРема 3. Пусть функция $f(x)$ такова, что $f(x)(x-a)^{\mu_{1}^{*}}$ абсолютно непрерывна на $[0, a] u[a, T], u f(0)=f(T)=0$. Тогда

$$
\lim _{N \rightarrow \infty} \max _{0 \leqslant x \leqslant T}\left|(x-a)^{\eta}\left(\frac{1}{2 \pi i} \int_{|\lambda|=r_{N}^{n}}\left(\int_{0}^{T} G(x, t, \lambda) f(t) d t\right) d \lambda+f(x)\right)\right|=0 .
$$

Отметим, что по теореме о вычетах интеграл в (4) равен частной сумме ряда Фурье функции $f(x)$ по собственным и присоединенньм функциям краевой задачи $\mathscr{L}$ и, следовательно, теорема 3 дает достаточные условия разложения $f(x)$ в равномерно сходящийся с весом ряд по собственным и присоединенным функциям задачи $\mathscr{L}$.

\section{СПИСОК ЦИТИРОВАННОЙ ЛИТЕРАТУРЫ}

1. Наймарк М. А. Линейные дифференциальные операторы. М.: Наука, 1969. 2. Костюченко А. Г., Саргсян И. С. Распределение собственных значений. Самосопряженные обыкновенные дифференциальные операторы. М.: Наука, 1979. 3. Birkhoff G. D. // Trans. Amer. Math. Soc. 1908. V. 9. № 4. Р. 373-397. 4. Тамаркин Я. Д. О некоторых общих задачах теории обыкновенных линейных дифференциальных уравнений. Петроград, 1917. 5. Хромов А. П. // Матем. сб. 1966. Т. 70 (112). №3. С. 310-329. 6. Шкаликов А. А. // Тр. семинара им. Петровского. 1983. T. 9. C. 190-229. 7. Beals R., Deift P., Tomei C. Direct and Inverse Scattering on the Line. Math. Surveys and Monographs. V. 28. Providence (R.I.): Amer. Math. Soc., 1988. 8. Yurko V. A. Inverse Problems for Differential Operators and their Applications. New York: Gordon and Breach, 2000. 9. Юрко В. А. // Дифференц. уравнения. 1992. Т. 28. № 8. С. 1355-1362. 10. Юрко В. А. // Матем. сб. 1995. Т. 186. №6. C. 133-160. 11. Yurko V. A. // Integral Transforms and Special Functions. 1997. V. 5. № 3-4. Р. 309-322. 12. Юрко В. А. // Матем. заметки. 1998. Т. 64. № 1. C. $143-156$.

Саратовский государственный университет им. Н. Г. Черньшевского 